\title{
Electronic and magnetic structure of $\mathrm{Ga}_{x} \mathrm{Fe}_{1-x}$ thin films
}

\author{
E. Arenholz, ${ }^{1}$ G. van der Laan, ${ }^{2}$ A. McClure, ${ }^{3}$ and Y. Idzerda ${ }^{3}$ \\ ${ }^{1}$ Advanced Light Source, Lawrence Berkeley National Laboratory, Berkeley, California 94720, USA \\ ${ }^{2}$ Diamond Light Source, Chilton, Didcot, Oxfordshire OX11 ODE, United Kingdom \\ ${ }^{3}$ Physics Department, Montana State University, Bozeman, Montana 59717, USA
}

(Dated: November 9, 2010)

\begin{abstract}
The electronic as well as magnetic properties of $\mathrm{Ga}_{x} \mathrm{Fe}_{1-x}$ films were studied by soft x-ray measurements. Using x-ray magnetic circular dichroism the Fe majority spin band was found to be completely filled for $x \approx 0.3$. With further enhanced Ga content, the Fe moment as well as the angular dependence of the x-ray magnetic linear dichroism decrease strongly, which we attribute to the formation of $\mathrm{D}_{3}$ precipitates. Moreover, the magnetocrystalline anisotropy drops significantly.

PACS numbers: 75.70.Ak, 75.70.Cn, 75.80.+q
\end{abstract}

In a magnetic material, the spin-orbit interaction, a purely relativistic effect between the electron spin and its orbital moment, couples the magnetic moments to the lattice. It is at the electronic origin of magnetocrystalline anisotropy, i.e., the energy associated with the (re)orientation of the magnetization relative to the lattice, as well as magnetostriction referring to the deformation of a magnetic material when subjected to an external magnetic field. In magnetostrictive thin films, the adaptation to new dimensions is inhibited by bonding to the substrate and the application of a magnetic field in the film plane does not result in a expansion or contraction along the field direction. Instead a magnetoelastic stress is created that leads to a change in magnetocrystalline anisotropy.

Here we study the magnetic as well as electronic properties of $\mathrm{Ga}_{x} \mathrm{Fe}_{1-x}$ films deposited on $\mathrm{GaAs}(001)$. In bulk form, $\mathrm{Ga}_{x} \mathrm{Fe}_{1-x}$ exhibits extraordinary magnetostrictive properties as function of composition implying a strong dependence of the underlying spin-orbit coupling on $x$ [1-3]. $\mathrm{Ga}_{x} \mathrm{Fe}_{1-x}$ thin films [4-6] are therefore an ideal candidate to study the composition dependence of the magnetic anisotropy and to correlate this with its electronic structure.

Using soft $\mathrm{x}$ ray absorption spectroscopy, we find that the magnetic as well as electronic properties of $\mathrm{Ga}_{x} \mathrm{Fe}_{1-x}$ films change significantly for Fe content near $x=0.3$. The holes in the Fe majority spin band disappear with increasing Ga content and the Fe majority spin band is completely filled for $x \approx 0.3$. With further enhanced Ga content, the Fe moment as well as the XMLD angular dependence decrease strongly, which we attribute to the formation of $\mathrm{D}_{3}$ precipitates.

For this study, $20 \mathrm{~nm}$ single crystalline $\mathrm{Ga}_{x} \mathrm{Fe}_{1-x}$ thin films, $0 \leq x \leq 0.6$, were deposited by molecular beam epitaxy as described in Ref. 5. X-ray absorption (XA) spectra were measured at ALS beamline 4.0.2 at ambient temperature with either $90 \%$ circularly or $100 \%$ linearly polarized x-rays in total electron yield mode by monitoring the sample drain current. External fields of $0.5 \mathrm{~T}$ were provided by an eight-pole electromagnet [7]. X-ray magnetic circular dichroism (XMCD) was derived from XA spectra measured with the magnetic field collinearly aligned to the incident $\mathrm{x}$-ray beam, which in turn impinged at an angle of $30^{\circ}$ to the sample surface. X-ray magnetic linear dichroism (XMLD) spectra are obtained as the difference between XA spectra in external fields parallel and perpendicular to the $\mathrm{x}$-ray polarization in normal incidence geometry.

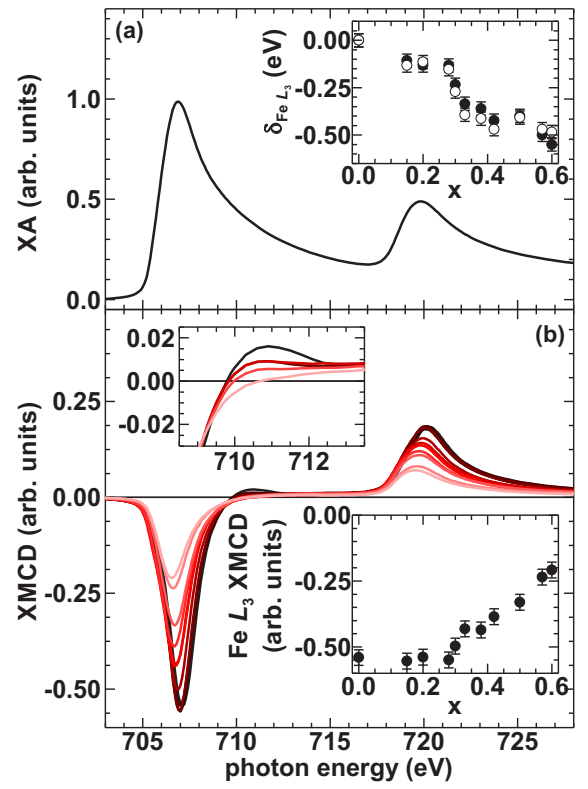

FIG. 1: (Color online) Fe $L_{3,2} \mathrm{XA}$ and XMCD spectra for $\mathrm{Ga}_{x} \mathrm{Fe}_{1-x}$. (a) Average XA spectrum. Inset: Change in photon energy of the $\mathrm{Fe} L_{3}$ edge derived from the XA (solid symbols) and XMCD spectra (open symbols). (b) XMCD spectra for varying Ga concentrations, $0 \leq x \leq 0.6$. Lighter colored lines correspond to data obtained from samples with higher Ga concentration. Top inset: Fe XMCD spectra near $711 \mathrm{eV}$ for $x \leq 0.28$. Bottom inset: Concentration dependence of the Fe $L_{3}$ XMCD signal.

Figure 1(a) shows the average of XA spectra obtained from $\mathrm{Ga}_{x} \mathrm{Fe}_{1-x}$ films with $0 \leq x \leq 0.6$. In the concentration dependence of the Fe $L_{3,2}$ XMCD shown in Fig. 1(b), we can distinguish two regions exhibiting distinct magnetic characteristics. For $x \leq 0.28$, the Fe $L_{3}$ 
XMCD signal - and with that to a good approximation the average Fe magnetic moment - is nearly constant and starts at $55 \%$ of the $\mathrm{Fe} L_{3}$ XA intensity in agreement with previous results for Fe films [8]. For $x \geq 0.3$, the Fe $L_{3}$ XMCD decreases almost linearly with Fe concentration. It is generally accepted that hybridization of the $\mathrm{Fe}$ and Ga states reduces the Fe magnetic moment [9-12]. Detailed calculations aimed at determining the concentration dependence of the Fe moment in different lattice structures and sites have been performed. However, there are significant variations in the results suggesting that an estimate of the average Fe moment as a function of Fe concentration using theory is not unambiguous at present.

The inset in Fig. 1(b) shows the spectral features at the high photon energy side of the $\mathrm{Fe} L_{3}$ edge for $x \leq 0.3$. For the pure Fe film, a small positive XMCD feature is observed at $\sim 711 \mathrm{eV}$, which can be ascribed to transitions from the $2 p$ core level to the unoccupied majority spin band $[13,14]$. The main negative Fe XMCD $L_{3}$ feature is due to transitions to the unoccupied minority spin band. The intensity of the XMCD feature at $\sim 711 \mathrm{eV}$ reduces with increasing Ga content, i.e., the majority spin band is gradually filled. For $x \approx 0.3$, the Fe $L_{3} \mathrm{XMCD}$ becomes fully negative indicating that the majority spin band is completely filled. We also see a shift in both the XA and XMCD $L_{3}$ peak energy to lower photon energy (inset Fig. 1(a)) consistent with band filling argument.

The occupation of majority spin states is closely correlated with the magnetostriction. Berger [15] showed that the magnetoelastic energy - and hence the magnetostriction - is determined by the electronic states located near the Fermi level, $E_{\mathrm{F}}$, and that it is directly proportional to the spin-orbit splitting. Consequently, the magnetostriction changes sign, i.e., zero magnetostriction is observed if $E_{\mathrm{F}}$ is located between the spin-orbit split bands.

Filling of the majority spin band also affects the magnetocrystalline anisotropy energy (MAE) $[16,17]$. Using second-order perturbation theory, i.e., considering the spin-orbit coupling to be small compared to energy differences caused by the crystalline electric field (CEF) and exchange interaction, one can distinguish two contributions to the MAE; those due to spin-conserved terms and those arising from spin-flip processes. An incompletely filled majority spin band allows low-energy spin-flip excitations near $E_{\mathrm{F}}$. When the majority spin band is full, however, such excitations require an energy in the order of the exchange interaction. Hence, unoccupied majority spin states enable spin-flip excitations, thereby changing the MAE. While the spin-flip contributions are usually not the dominant contribution in the total MAE, they will lead to a change in MAE $[12,16,17]$.

Previous studies on transition metal and lanthanide oxide thin films [18-21] have shown that the angular dependence of the XMLD provides a sensitive measure of

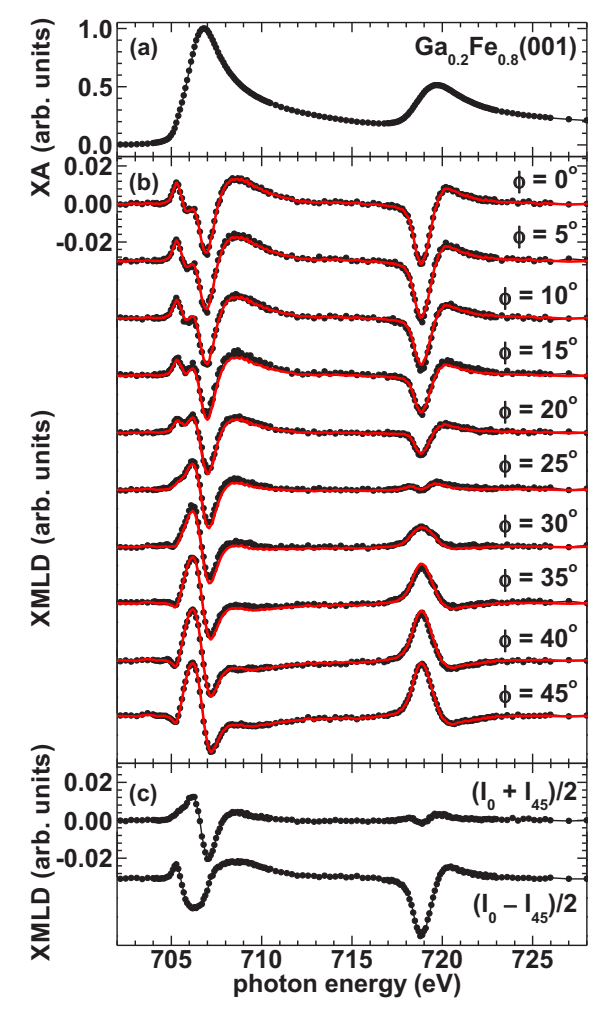

FIG. 2: (Color online) Fe $L_{3,2} \mathrm{XA}$ and XMLD spectra for $\mathrm{Ga}_{0.2} \mathrm{Fe}_{0.8}$. (a) XA spectrum. (b) XMLD spectra for varying angle $\phi$ between the x-ray polarization and the [100] direction. The (black) symbols indicate the experimental data while the (red) lines represent the results of the model discussed in the text. (c) Derived XMLD spectra $\frac{1}{2}\left(I_{0}+I_{45}\right)$ and $\frac{1}{2}\left(I_{0}-I_{45}\right)$, where $I_{0}$ and $I_{45}$ refer to the XMLD spectra obtained for $\phi=0^{\circ}$ and $\phi=45^{\circ}$, respectively.

the CEF symmetry and strength as well as magnetic and electronic characteristics of the absorber. We determined the Fe $L_{3,2}$ XMLD spectra, $I_{\mathrm{XMLD}}(\phi)$, for varying angle $\phi$ between the linear x-ray polarization, $\mathbf{E}$, and the [100] lattice direction of $\mathrm{Ga}_{0.2} \mathrm{Fe}_{0.8}$. The XMLD spectrum is defined here as the difference between XA spectra with $\mathbf{H}$ parallel and perpendicular to $\mathbf{E}$, i.e., at angles $\phi$ and $\phi+90^{\circ}$ to the [100] direction,

$$
I_{\mathrm{XMLD}}(\phi)=I_{\mathrm{XA}}\left(\mathbf{H}_{\phi}, \mathbf{E}_{\phi}\right)-I_{\mathrm{XA}}\left(\mathbf{H}_{\phi+90^{\circ}}, \mathbf{E}_{\phi}\right),
$$

The averaged Fe XA signal is shown in Fig. 2(a) and the XMLD angular dependence for $0^{\circ} \leq \phi \leq 45^{\circ}$ in Fig. 2(b). A pronounced variation of the Fe XMLD signal with $\phi$ is observed. In lattices with cubic symmetry, the angular dependent XMLD can be very well described by a linear combination of two fundamental spectra accounting for the XA photon energy dependence in selected orientations [19-21]. Their relative contributions are determined by the symmetry of the experimental geometry. In the (001) plane, the XMLD angular dependence is described by

$$
I_{\mathrm{XMLD}}(\phi)=\frac{1}{2}\left[\left(I_{0}+I_{45}\right)+\left(I_{0}-I_{45}\right) \cos 4 \phi\right],
$$


where $I_{0}$ and $I_{45}$ refer to the XMLD spectra obtained with the x-ray polarization $\mathbf{E}$ at $0^{\circ}$ and $45^{\circ}$ to the [100] direction, i.e., along the [100] and [110] directions, respectively. Equation (2) allows us to separate the angular dependent XMLD into an angle-independent or isotropic contribution to the XMLD signal, $\frac{1}{2}\left(I_{0}+I_{45}\right)$, and an angular part or anisotropic contribution, $\frac{1}{2}\left(I_{0}-I_{45}\right)$, which gives the difference in the XMLD along two principal directions. Figure 2(c) shows the experimental results for $\frac{1}{2}\left(I_{0}+I_{45}\right)$ and $\frac{1}{2}\left(I_{0}-I_{45}\right)$. Applying Eq. (2) and using the results from Fig. 2(c), leads to the calculated spectra shown in direct comparison with the experimental data in Fig. 2(b). The agreement is excellent indicating that the approach of separating the anisotropy due to CEF from the detailed electronic structure of the excited atom originally developed for transition metal oxides is also valid for itinerant electron systems such as $\mathrm{Fe}$ and $\mathrm{Ga}_{x} \mathrm{Fe}_{1-x}$.

We determined the concentration dependence of the Fe XMLD spectra $I_{0}$ and $I_{45}$ for $0 \leq x \leq 0.6$. The experimental results for $\frac{1}{2}\left(I_{0}+I_{45}\right)$ and $\frac{1}{2}\left(\bar{I}_{0}-I_{45}\right)$ are shown in Fig. 3(a). Both contributions decrease significantly with increasing Ga content. The XMLD signal was shown to be proportional to the square of the magnetic moment [18]. To account for the observed change in Fe moment with Ga content [see Fig. 1(b)], we normalized the XMLD signals to the square of the Fe $L_{3}$ XMCD signal that to good approximation is a measure for the Fe magnetic moment. The results are shown in Fig. 3(b) for the isotropic XMLD contribution and we find a constant $\frac{1}{2}\left(I_{0}+I_{45}\right)$ signal for the entire concentration range. We conclude that the magnitude of the isotropic XMLD signal $\frac{1}{2}\left(I_{0}+I_{45}\right)$ is solely determined by the magnitude of the Fe magnetic moment. Small changes in the spectral shape are attributed to changes in the detailed electronic structure also seen in the $\mathrm{Fe}$ $\mathrm{XMCD}$ as discussed above. Using the same normalization for the angle dependent signal $\frac{1}{2}\left(I_{0}-I_{45}\right)$ leads to a still strongly decreasing XMLD signal with decreasing $\mathrm{Fe}$ concentration. As shown in Figure 3(c) normalizing $\frac{1}{2}\left(I_{0}-I_{45}\right)$ to the Fe concentration, i.e., scaling it by $(1-x)^{-1}$ as well as the square of the average Fe moment, leads to a constant anisotropic XMLD signal for $x \leq 0.28$ as well as for $x \geq 0.3$. This indicates that additional factors beyond the concentration dependence of the Fe moment play a role in determining the XMLD angular dependence. One possible explanation is that for $x \geq 0.3$ a volume fraction of the $\mathrm{Ga}_{x} \mathrm{Fe}_{1-x}$ film consists of a phase where the Fe XMLD signal does not exhibit an angular dependence, i.e., is highly isotropic. The volume fraction of $\mathrm{Fe}$ atoms in this phase increases with decreasing Fe content to account for the decrease in $\frac{1}{2}\left(I_{0}-I_{45}\right)$ with $x$.

In case of a completely disordered or isotropic system, no angular dependence is expected, i.e., $I_{0}-I_{45}=0$. Our reflection high energy electron diffraction (RHEED) measurements during film deposition indicate that the structural quality of the $\mathrm{Ga}_{x} \mathrm{Fe}_{1-x}$ does not dependent significantly on the Fe concentration, i.e., structural disorder cannot account for the reduction of $\frac{1}{2}\left(I_{0}-I_{45}\right)$ with increasing Ga content.

In epitaxial $\mathrm{EuO}$ thin films, the $\mathrm{Eu} M_{5,4}$ XMLD shows only a very small angular dependence, which could be explained by the nearly perfect spherical shape of the half filled Eu $4 f$ shell [21]. For $\mathrm{Ga}_{x} \mathrm{Fe}_{1-x}$, Lei et al. [10] calculated the anisotropy of the $\mathrm{Fe} 3 d$ orbitals for $\mathrm{D}_{3}$, B2-like, and $\mathrm{L} 1_{2}$ lattice structures [11]. They showed that the $\mathrm{D} 0_{3}$ structure has an almost isotropic $3 d$ charge density and that there is hardly any difference between the two $\mathrm{Fe}$ sites in this lattice structure. By contrast, a pronounced anisotropy was found for the other two phases. This suggest that $\mathrm{Fe}$ in local $\mathrm{D}_{3}$ environments could be responsible for the reduction in the Fe XMLD angular dependence with decreasing Fe concentration. Moreover, van't Erve et al. [4] concluded from RHEED measurements during deposition of $\mathrm{Ga}_{x} \mathrm{Fe}_{1-x}$ films on AlGaAs/GaAs quantum well structures that the $\mathrm{D}_{3}$ phase is present for $x \geq 0.25$. These results lead us to conclude that with increasing Ga content the $\mathrm{D}_{3}$ phase develops and the fraction of $\mathrm{Fe}$ in these $\mathrm{D}_{3}$ precipitates increases with decreasing Fe concentration. This accounts for the change in the angular dependent XMLD with Fe concentration $1-x$.

Having discussed concentration dependence of the magnitude of the XMLD signal we now consider the variation of the Fe XMLD spectral shape with Ga concentration. The XMLD spectral shape is influenced by the CEF symmetry and strength as well as the magnetic and electronic characteristics of the absorber. In case of $\mathrm{Ga}_{x} \mathrm{Fe}_{1-x}$ alloys, the CEF created by the nearest neighbors of the Fe absorber is not expected to be very sensitive to change in the elemental species of between Fe and Ga for identical structural parameters. As a consequence the spectral shape of the Fe XMLD signal is expected to be the same for $b c c$ Fe and a disordered $\mathrm{Ga}_{x} \mathrm{Fe}_{1-x}$ alloy with the same $b c c$ structure, i.e. $\mathrm{Ga}_{x} \mathrm{Fe}_{1-x}$ in A2 phase. The experiments show that the spectral shape of $\frac{1}{2}\left(I_{0}-I_{45}\right)$ is the same for all Ga concentrations $x$, i.e. identical to pure $b c c$ - $\mathrm{Fe}(x=0)$. Therefore, we attribute the $\frac{1}{2}\left(I_{0}-I_{45}\right)$ signal to Fe atoms in the A2 phase.

To determine the impact of the concentration dependence of the Fe electronic structure in $\mathrm{Ga}_{x} \mathrm{Fe}_{1-x}$ films on their magnetic anisotropy, we applied the XMLD sum rules to the experimental results for $\frac{1}{2}\left(I_{0}+I_{45}\right)$ and $\frac{1}{2}\left(I_{0}-I_{45}\right)[22]$. The integrated intensities, $A_{L_{3}}$ and $A_{L_{2}}$ of the Fe $L_{3}$ and $L_{2}$ XMLD signal, respectively, were determined using the experimental results for $\frac{1}{2}\left(I_{0}+I_{45}\right)$ and $\frac{1}{2}\left(I_{0}-I_{45}\right)$ shown in Fig. $3(\mathrm{a})$. The integrated signal $A=A_{L_{3}}-2 A_{L_{2}}$ is related via the sum rule to the anisotropic spin-orbit interaction, which in turn is a measure of the magnetocrystalline anisotropy energy (MAE). Previous experiments suggest that it is not straightforward to directly relate the integrated signals to the absolute value of the MAE [23]. However, 


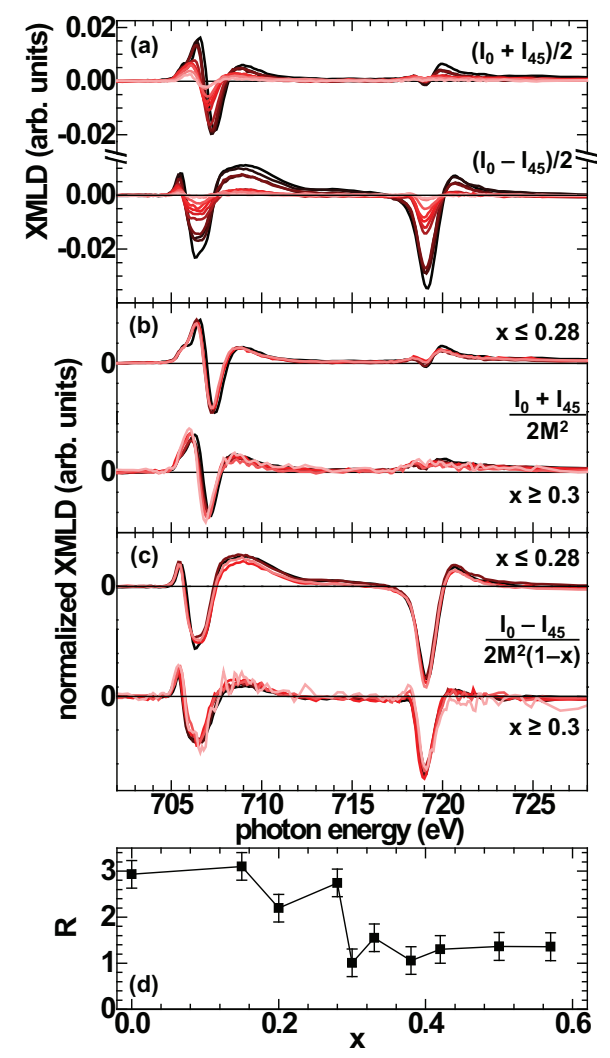

FIG. 3: (Color online) Concentration dependence of the $\mathrm{Fe}$ $L_{3,2}$ XMLD signal in $\mathrm{Ga}_{x} \mathrm{Fe}_{1-x}$. The results for $x=0$ are shown in black while lighter colored lines correspond to data obtained from samples with higher Ga concentration. (a) $\frac{1}{2}\left(I_{0}+I_{45}\right)$ (top) and $\frac{1}{2}\left(I_{0}-I_{45}\right)$ (bottom) for $x \leq 0.28$. (b) $\frac{1}{2}\left(I_{0}+I_{45}\right)$ normalized to the Fe $L_{3}$ XMCD signal for $x \geq 0.3$ (top) and for $x \geq 0.3$ (bottom). (c) $\frac{1}{2}\left(I_{0}-I_{45}\right)$ normalized to the square of the Fe $L_{3}$ XMCD signal and the Fe concentration, $1-x$, for $x \leq 0.28$ (top) and for $x \geq 0.3$ (bottom). (d) Concentration dependence of the ratio $R=A_{I_{0}-I_{45}} / A_{I_{0}+I_{45}}$, see text.

here we measure a material where the composition gradually changes and therefore the trend in the MAE should be qualitatively correct. Moreover, by evaluating the ratio $R=A_{I_{0}-I_{45}} / A_{I_{0}+I_{45}}$ we eliminate the impact of factors such as degree of magnetization and polarization, $3 d$ localization, number of holes, etc. The dimensionless quantity $R$ is a measure for the magnetic anisotropy between the [100] and [110] directions. The results shown in Fig. 3(d) indicate that the MAE is approximately constant for $x \leq 0.28$ and abruptly drops by a factor two with further decreasing Fe concentration. The MAE and magnetostriction are sensitive properties of the crystal structure, band filling, and $E_{\mathrm{F}}$ position. Our results indicate that for $x>0.28$, i.e., for concentrations with the majority spin band filled, the MAE is much reduced. As mentioned above, spin-flips inhibited by the filled majority spin band are a possible origin of the reduced MAE, however, they do not necessarily form the main cause.

We summarize that the magnetic as well as electronic properties of $\mathrm{Ga}_{x} \mathrm{Fe}_{1-x}$ films change significantly for $\mathrm{Fe}$ content near $x=0.3$. The holes in the Fe majority spin band disappear with increasing $\mathrm{Ga}$ content and the Fe majority spin band is completely filled for $x \approx 0.3$. With further enhanced Ga content, the Fe moment as well as the XMLD angular dependence decrease strongly, which we attribute to the formation of $\mathrm{D}_{3}$ precipitates. In bulk $\mathrm{Ga}_{x} \mathrm{Fe}_{1-x}$, the formation of $\mathrm{D}_{3}$ precipitates has been observed near $x=0.19$ and is associated with a maximum in magnetostriction. A second maximum of the magnetostriction is observed near $x=0.28$ attributed to a softening of the shear modulus, coinciding with our observation of Fe majority spin band filling. Further experimental and theoretical studies are certainly warranted to explain the differences in structure, magnetic, and electronic between bulk and thin film $\mathrm{Ga}_{x} \mathrm{Fe}_{1-x}$ samples.

Supported by the U.S. Department of Energy under Contract No. DE-AC02-05CH11231 and grant \#w911NF-08-1-0325 from the Army Research Office.

[1] A. E. Clark, et al., IEEE Trans. Magn. 36, 3238 (2000).

[2] A. E. Clark, et al., J. Appl. Phys. 93, 8621 (2003).

[3] M. Wuttig, L. Dai, and J. Cullen, Appl. Phys. Lett. 80, 1135 (2002).

[4] O. M. J. vant Erve, et al., Appl. Phys. Lett. 91, 122515 (2007).

[5] A. McClure, et al., J. Appl. Phys. 105, 07 A938 (2009).

[6] A. McClure, H. Li, and Y. U. Idzerda, J. Appl. Phys. 107, 09A924 (2010).

[7] E. Arenholz and S. O. Prestemon, Rev. Sci. Instrum. 76, 083908 (2005).

[8] C. T. Chen, et al., Phys. Rev. Lett. 75, 152 (1995).

[9] J. M. Hill, et al., Phys. Rev. B 77, 014430 (2008).

[10] Z. Lei, J. Cheng-Bao, S. Jia-Xiang, and X. Hui-Bin, Chinese Phys. B 18, 1647 (2009).

[11] T. Khmelevska, S. Khmelevskyi, and P. Mohn, J. Appl. Phys. 103, 073911 (2008).

[12] S. C. Hong, W. S. Yun, and R. Wu, Phys. Rev. B 79, 054419 (2009).

[13] G. van der Laan, Phys. Rev. B 55, 8086 (1997).

[14] G. van der Laan, J. Phys.:Condens. Matter 9, L259 (1997).

[15] L. Berger, Physica 91B, 31 (1977).

[16] D. S. Wang, R. Wu, and A. J. Freeman, Phys. Rev. B 47, 14932 (1993).

[17] G. van der Laan, J. Phys.: Condens. Matter 10, 3239 (1998).

[18] A. A. Freeman, et al., Phys. Rev. B 73, 233303 (2006).

[19] E. Arenholz, G. van der Laan, R. V. Chopdekar, and Y. Suzuki, Phys. Rev. B 74, 094407 (2006).

[20] E. Arenholz, G. van der Laan, R. V. Chopdekar, and Y. Suzuki, Phys. Rev. Lett. 98, 197201 (2007).

[21] G. van der Laan, E. Arenholz, A. Schmehl, and D. G. Schlom, Phys. Rev. Lett. 100, 067403 (2008).

[22] G. van der Laan, Phys. Rev. Lett. 82, 640 (1999).

[23] S.S. Dhesi, G. van der Laan, E. Dudzik, and A.B. Shick, Phys. Rev. Lett. 87, 067201 (2001). 